\title{
Investigation of the primary breakup length and instability of non-Newtonian viscoelastic liquid jets
}

\author{
H Khodayari', F Ommi', Z Saboohi ${ }^{2 *}$ \\ 1. Tarbiat Modares University, Tehran, Iran \\ 2. Aerospace Research Institute (ARI), Ministry of Science, \\ Research, and Technology, Tehran, Iran
}

\begin{abstract}
The temporal instability and primary breakup length of a non-Newtonian viscoelastic liquid jet moving in an inviscid gaseous environment were carried out by solving a set of linearized Navier-Stokes equations and employing the linear viscoelastic model, respectively. The dimensionless dispersion equation that governs the instability was derived and solved by a numerical method. The effects of fluid properties on the instability and primary breakup length of viscoelastic liquid jets were carried out. It could be seen that by increasing the growth rate, the instability range and the primary breakup length of the viscoelastic liquid jets could result in an increase in the liquid Weber number and the ratio of gas to liquid density. Moreover, the significant findings are that an increase in the time constant ratio, and also the Ohnesorge number reduced both of the growth rates of disturbances and primary breakup length. Though, increasing the elasticity number resulted in a higher growth rate of disturbances and enhanced the breakup mechanism.
\end{abstract}

\section{INTRODUCTION}

When a liquid jet exits from an atomizer, it becomes unstable. The amplitude of the disturbances on the liquid-gas interface grows and leads to the breakup of the liquid sheet into ligaments and then into droplets. This multiphysics process is called atomization and is used in several industrial processes: fuel injection in combustion processes, chemical combustion, spray drying, evaporative cooling, agricultural sprays, and medicine. Since the efficiency and quality of production are strongly dependent on these mechanisms, it is essential to understand the physics of instability and the breakup of liquid jets and sheets [1]. Rayleigh [2] was the first to address the linear instability of an inviscid cylindrical liquid column in the absence of surrounding gas. Liquid viscosity and surrounding gas pressure were considered in the study done by Weber [3]. A review of the linear instability theories for liquid jets is given by Lefebvre [4], Lin [5] and Sirignano and Mehring [6]. Reitz and Bracco [7] concluded that the combination of aerodynamic interaction theory and nozzle geometry was adequate to explain their experimental results. It is generally acknowledged that the aerodynamic interactions between the liquid and the ambient gas play a significant role in the breakup of the liquid jet. Their aerodynamic interactions enhance the unstable wave growth rate on the liquid surface, and then these unstable waves cause the liquid jet to break into ligaments and then into droplets

*Corresponding Author: saboohi@ari.ac.ir 
[8]. The instability and breakup of inviscid liquid sheets of uniform thickness in an inviscid gas environment were analyzed by Squire [9] and Hagerty and Shea [10]. Their results showed that the surface tension forces always tend to dampen out any protuberances, and the aerodynamic forces are responsible for the instability of inviscid sheets. The characteristics of instability and breakup of Newtonian liquid sheets in an inviscid gaseous medium were analyzed by $\mathrm{Li}$ and Tankin [11]. Li and Tankin's results showed that for Newtonian fluids, the surface tension always opposes, while the relative motion between the sheet and gas favors the onset and development of instability. Since many fluids used in atomization applications in practical fields are non-Newtonian liquids, it is significant to study the growth of instability in non-Newtonian liquid jets [12-15]. Yarin [16] studied in detail the instability and breakup mechanisms of non-Newtonian liquid jets. Rallison and Hinch [17] considered the linearized inertial instability of the parallel shear flow of a viscoelastic liquid. Keller et al. [18] explored the spatial instability of a circular cylindrical jet of liquid in ambient air. Gordon et al. [19] studied the instability of several nonNewtonian fluid jets under the influence of externally controlled disturbances and then investigated the breakup patterns of each fluid. Brenn et al. [20] investigated the temporal instability behavior of non-Newtonian liquid jets moving in an inviscid gaseous environment for axisymmetrical disturbances, and then gained the corresponding dispersion relation between the wave growth rate and the wave number. According to their report, the linear stability analysis showed that a jet of a viscoelastic fluid exhibited a more significant growth rate of axisymmetric disturbances than a jet of a Newtonian fluid with the same Ohnesorge number. The results indicated that non-Newtonian liquid jets were more unstable than Newtonian jets.

The objective of the present work was to derive a distribution relation by means of linear stability analysis for non-Newtonian viscoelastic liquid jets under the action of surface tension and aerodynamic forces, as well as to investigate and analyze its predictions of the jet characteristics. The results of the present model will be compared with other theories of jet instability. The influence of non-dimensional parameters on the stability and primary breakup of the viscoelastic liquid jet will also be discussed.

\section{FORMULATION FOR LINEAR INSTABILITY ANALYSIS}

Consider an incompressible viscoelastic liquid jet of density $\rho$, surface tension $\sigma$, and radius a moving at constant velocity $\bar{U}$ through an inviscid and incompressible gas of density $\rho_{g}$. The governing equations are written in a cylindrical coordinate system, with the z-axis along the centerline of the jet, and the $r$ axis is normal to the liquid jet with its origin located at the axis of symmetry. The axisymmetric disturbances on the surface of the liquid jet are considered in this study. Figure 1 shows a schematic diagram of a moving liquid jet and the coordinate system.

The governing equations of the liquid motion in a jet are the conservation laws of mass and momentum, as given in Equation (1) and (2).

$$
\begin{gathered}
\frac{\partial \rho}{\partial t}+\nabla \cdot \rho v=0, \\
\rho\left(\frac{\partial}{\partial t}+v \cdot \nabla\right) v=-\nabla \cdot \pi+\rho g,
\end{gathered}
$$

where $\mathrm{t}$ is the time, $\mathrm{v}$ is the liquid velocity vector, $\mathrm{g}$ is the gravitational acceleration vector, and $\pi$ is the total stress tensor of the liquid, which is given by Equation (3). 


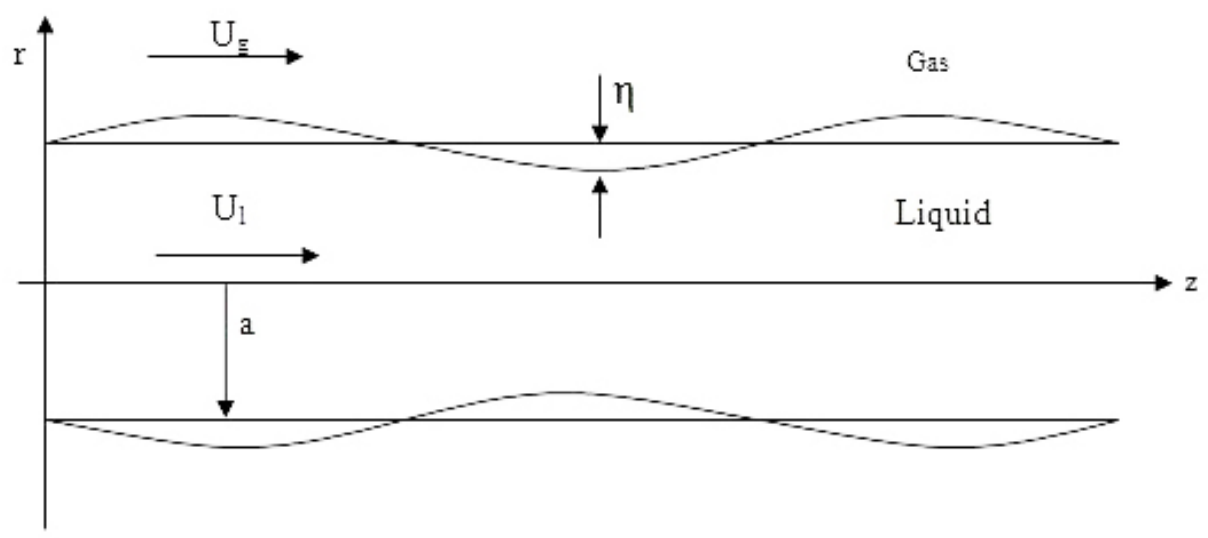

Figure 1: Schematic diagram of a liquid jet with axisymmetrical surface disturbances and the laboratory-fixed coordinate system $r, z$.

$$
\pi=p \delta+\tau
$$

where $\mathrm{p}$ is the pressure of the liquid due to the disturbance, $\tau$ is the extra stress tensor of the liquid, and $\delta$ is the unit tensor.

The viscoelastic properties of the fluid are described using the corotational Oldroyd eightconstant model which has the general constitutive equation in the objective reference frames in the form of Equation (4).

$$
\begin{gathered}
\tau+\lambda_{1} \frac{D \tau}{D t}+\frac{1}{2} \mu_{0}(\operatorname{tr} \tau) \dot{\gamma}-\frac{1}{2} \mu_{1}\{\tau \cdot \dot{\gamma}+\dot{\gamma} \cdot \tau\}+\frac{1}{2} v_{1}(\tau: \dot{\gamma}) \delta \\
=-\eta_{0}\left[\dot{\gamma}+\lambda_{2} \frac{D \dot{\gamma}}{D t}-\mu_{2}\{\dot{\gamma} \cdot \dot{\gamma}\}+\frac{1}{2} v_{2}(\dot{\gamma}: \dot{\gamma}) \delta\right]
\end{gathered}
$$

where $\lambda_{1}$ is the stress relaxation time, $\lambda_{2}$ is the deformation retardation time of the liquid, $\delta$ is the unit tensor and $\mu_{0}$ is the zero shear viscosity.

The rate of strain tensor $\dot{\gamma}$ and the vorticity tensor $\omega$ are defined by Equation (5) and Equation (6).

$$
\begin{aligned}
& \dot{\gamma}=\nabla v+(\nabla v)^{T} \\
& \omega=\nabla v-(\nabla v)^{T}
\end{aligned}
$$

$\mathrm{D} / \mathrm{Dt}$ is defined as the corotational derivative and given by Equation (7) and Equation (8).

$$
\frac{D \tau}{D t}=\frac{\partial \tau}{\partial t}+(v \cdot \nabla) \tau+\frac{1}{2}\{\omega \cdot \tau-\tau . \omega\}
$$




$$
\frac{D \dot{\gamma}}{D t}=\frac{\partial \dot{\gamma}}{\partial t}+(v \cdot \nabla) \dot{\gamma}+\frac{1}{2}\{\omega \cdot \dot{\gamma}-\dot{\gamma} \cdot \omega\}
$$

Similarly the governing equations for the inviscid and incompressible gas are expressed as Equation (9) and Equation (10).

$$
\begin{gathered}
\nabla \cdot u_{g}=0 \\
\rho_{g}\left(\frac{\partial}{\partial t}+v_{g} \cdot \nabla\right) v_{g}=-\nabla \cdot p_{g}
\end{gathered}
$$

where $\rho_{g}$ is the gas density, $u_{g}$ is gas velocity vector and $p_{g}$ is the gas pressure.

For linear stability analysis of the liquid jets, the dependent variables for the pressures and velocities should be presented as the summation of the initial steady state and the unsteady disturbances terms (Equation (11) and Equation (12)). When the jet is disturbed, the gas-liquid interface will displace from the equilibrium position $r=a$, such that the disturbed flow field becomes as Equation (13).

$$
\begin{gathered}
U_{j}=\bar{U}_{j}+u_{j} \quad j: g, l \\
p_{j}=\bar{p}_{j}+p_{j} \\
r=a+\xi
\end{gathered}
$$

where $r=a$ is the equilibrium position of the jet surface, i.e., the position without disturbances, and $\xi$ is the displacement of a point on the surface.

The following linearized equations (Equations (14-16)) are obtained after neglecting the nonlinear terms and gravitational effects.

$$
\begin{gathered}
\nabla \cdot v=0 \\
\rho\left(\frac{\partial}{\partial t}+\bar{U} \frac{\partial}{\partial x}\right) v=-\nabla \cdot(p \delta+\tau) \\
\tau+\lambda_{1}\left(\frac{\partial}{\partial t}+\bar{U} \frac{\partial}{\partial x}\right) \tau=-\eta_{0}\left[\dot{\gamma}+\lambda_{2}\left(\frac{\partial}{\partial t}+U \frac{\partial}{\partial x}\right) \dot{\gamma}\right]
\end{gathered}
$$

where $\eta_{0}$ is described as the zero shear viscosity of the fluid and the ratio of $\lambda_{2} / \lambda_{1}$ is defined as time constant ratio.

The disturbances are assumed in the forms of normal mode as Equation (17).

$$
(v, \tau, \dot{\gamma}, p, \xi)=\left(\hat{v}(r), \hat{\tau}(r), \hat{\gamma}(r), \hat{p}(r), \xi_{0}\right) e^{i k z+\alpha t}
$$

where ${ }^{\wedge}$ indicates the disturbance amplitude, which is presumed to be independent of $t$ and $\mathrm{z}$ but dependent only to position r. $\xi_{0}$ is the initial amplitude of the disturbance, which is 
taken to be much smaller than the radius of the liquid jet. The wave number of disturbance $\mathrm{k}$ is related to the disturbance wave length by $k=\frac{2 \pi}{\lambda}$. Also, $\alpha$ is the complex frequency where the real part $\alpha_{r}$ represents the rate of growth of disturbances with time, and the imaginary part $\alpha_{i}$ is $2 \pi$ times the disturbance frequency.

The flow field solutions of the above governing equations for both the liquid and gas phase have to satisfy the kinematic and dynamic boundary conditions at the gas-liquid interface, which can be taken at $r=a$. The linearized kinematic boundary condition to the first order can be expressed as Equation (18) and Equation (19).

$$
\begin{array}{cc}
v_{r, l}=\frac{\partial \xi}{\partial t}+\bar{U} \frac{\partial \xi}{\partial z}, & r=a \\
v_{r, g}=\frac{\partial \xi}{\partial t} & r=a
\end{array}
$$

And the dynamic boundary conditions require that can be expressed as Equation (20) and Equation (21).

$$
\begin{array}{cc}
\tau_{r z}=-\eta(\alpha)\left(\frac{\partial v_{z}}{\partial r}+\frac{\partial v_{r}}{\partial z}\right)=0, & r=a \\
\pi_{r r}-\pi_{G, r r}+p_{\sigma}=0, & r=a
\end{array}
$$

where $\pi_{r r}$ is the liquid normal stress, $\pi_{G, r r}$ is the gas normal stress, and $p_{\sigma}$ is the pressure induced by the surface tension and it can be expressed as Equation (22).

$$
p_{\sigma} \approx-\frac{\sigma}{a^{2}}\left(\eta+a^{2} \frac{\partial^{2} \eta}{\partial z^{2}}\right)
$$

Moreover, the velocity and pressure must be finite at $r=a$ for liquid. Note that Equation (4) is linearized as shown in Equation (23).

$$
\hat{\tau}=\eta(\omega) \hat{\gamma}
$$

where the effective viscosity $\eta(\omega)$ can be expressed as Equation (24).

$$
\eta(\omega)=\eta_{0} \frac{1+\lambda_{2}(\omega+i k u)}{1+\lambda_{2}(\omega+i k u)}
$$

Substituting Equation (23) into Equation (15) and expressing the continuity and momentum equations in a suitable component will yield the following governing equations.

$$
\frac{\partial u}{\partial z}+\frac{1}{r} \frac{\partial v}{\partial r}=0
$$




$$
\begin{gathered}
\rho\left(\frac{\partial u}{\partial t}+U \frac{\partial u}{\partial z}\right)=-\frac{\partial p}{\partial z}+\eta(\omega)\left(2 i k \frac{\partial u}{\partial z}+\frac{1}{r}\left(\frac{\partial v}{\partial z}+\frac{\partial u}{\partial r}\right)+i k \frac{\partial v}{\partial r}+\frac{\partial^{2} u}{\partial r^{2}}\right) \\
\rho\left(\frac{\partial v}{\partial t}+U \frac{\partial v}{\partial z}\right)=-\frac{\partial p}{\partial r}+\eta(\omega)\left(-\left(k^{2}+\frac{1}{r^{2}}\right) v+\frac{1}{r} \frac{\partial v}{\partial r}+\frac{\partial^{2} v}{\partial r^{2}}\right)
\end{gathered}
$$

The substitution of Equation (17) into the above equations of motion yields ordinary differential equations for the amplitude functions which are dependent on the radial coordinate only.

$$
\begin{gathered}
i k \hat{u}+\frac{\hat{v}}{r}+\frac{d \hat{v}}{d r}=0 \\
(\omega+i k U) \hat{u}=-\frac{(i k) \dot{p}}{\rho}+\frac{\eta(\omega)}{\rho}\left[-2 k^{2} \hat{u}+i k\left(\frac{\hat{v}}{r}+\frac{d \hat{v}}{d r}\right)+\frac{1}{r} \frac{d \hat{u}}{d r}+\frac{d^{2} \hat{u}}{d r^{2}}\right] \\
(\omega+i k U) \hat{v}=-\frac{1}{\rho} \frac{d \dot{p}}{d r}+\frac{\eta(\omega)}{\rho}\left[-\left(k^{2}+\frac{1}{r^{2}}\right) \hat{v}+\frac{1}{r} \frac{d \hat{v}}{d r}+\frac{d^{2} \hat{v}}{d r^{2}}\right]
\end{gathered}
$$

The application of the boundary conditions allows for the calculation of the integration constants which appear in the solutions of these differential equations. The final forms of the velocity and pressure profiles in the liquid jet read as follows:

$$
\begin{array}{ll}
v_{r}=\left[\frac{l^{2}+k^{2}}{I_{1}(k a)} I_{1}(k r)-\frac{2 k^{2}}{I_{1}(l a)} I_{1}(l r)\right] \frac{\eta(\alpha)}{\rho} \xi_{0} e^{i k z+\alpha t} & r \leq a \\
v_{z}=i\left[\frac{l^{2}+k^{2}}{I_{1}(k a)} I_{0}(k r)-\frac{2 k l}{I_{1}(l a)} I_{0}(l r)\right] \frac{\eta(\alpha)}{\rho} \xi_{0} e^{i k z+\alpha t} & r \leq a \\
p=-\frac{\rho(\alpha+i k \bar{U})^{2}\left(l^{2}+k^{2}\right)}{k\left(l^{2}-k^{2}\right) I_{1}(k a)} I_{0}(k r) \eta(\alpha) \xi_{0} e^{i k z+\alpha t} & r \leq a
\end{array}
$$

where

$$
\begin{gathered}
l^{2}=k^{2}+\frac{\rho(\alpha+i k \bar{U})}{\eta(\alpha)} \\
\eta(\alpha)=\eta_{0} \frac{1+\lambda_{2}(\alpha+i k \bar{U})}{1+\lambda_{1}(\alpha+i k \bar{U})}
\end{gathered}
$$

And $I(k r)$ is the modified Bessel function of the first kind. Then, the normal stress in the liquid jet can be obtained.

$$
\pi_{r r}=p+2 \eta(\omega) \frac{\partial v}{\partial r}
$$

In the present analysis, the gas around the moving liquid jet is assumed to be inviscid and incompressible; it moves at a velocity $\bar{U}_{G}$ in the same direction as the flow of the liquid jet. 
Similar to the liquid phase, the governing equations for the gas phase are expressed in a linearized form as follows:

$$
\begin{gathered}
\frac{1}{r} \frac{\partial}{\partial r}\left(r v_{r, G}\right)+\frac{\partial v_{z, G}}{\partial z}=0 \\
\rho_{G}\left(\frac{\partial v_{r, G}}{\partial t}+\bar{U}_{G} \frac{\partial v_{r, G}}{\partial z}\right)=-\frac{\partial p_{G}}{\partial r} \\
\rho_{G}\left(\frac{\partial v_{z, G}}{\partial t}+\bar{U}_{G} \frac{\partial v_{z, G}}{\partial z}\right)=-\frac{\partial p_{G}}{\partial z}
\end{gathered}
$$

where Equation (37) is the gas phase continuity equation, and Equation (38) and Equation (39) are the gas phase momentum equations. The linearized boundary conditions for the gas phase are:

$$
\begin{array}{cc}
v_{r, G}=\frac{\partial \xi}{\partial t}+\bar{U}_{G} \frac{\partial \xi}{\partial z} & r=a \\
v_{r, G}=0 & r \rightarrow \infty
\end{array}
$$

Similar to the governing differential equations for the liquid phase, the solutions for the velocity and pressure profiles in the gas phase are sought in the following form:

$$
\left(u_{g}, v_{g}, \dot{p}_{g}, \xi\right)=\left(\hat{u}_{g}(r), \hat{v}_{g}(r), \hat{p}_{g}(r), \xi_{0}\right) e^{i k z+\alpha t}
$$

The same calculation as for the liquid phase leads to the final form of the profiles of the two velocity components and the pressure in the gas phase. The equations read

$$
\begin{array}{ll}
v_{r, G}=\frac{\alpha+i k \bar{U}_{G}}{K_{0}^{\prime}(k a)} K_{0}^{\prime}(k r) \xi_{0} e^{i k z+\alpha t} & r \geq a \\
v_{z, G}=i \frac{\alpha+i k \bar{U}_{G}}{K_{0}^{\prime}(k a)} K_{0}(k r) \xi_{0} e^{i k z+\alpha t} & r \geq a \\
p_{G}=-\frac{\rho_{G}}{k} \frac{\left(\alpha+i k \bar{U}_{G}\right)^{2}}{K_{0}^{\prime}(k a)} K_{0}(k r) \xi_{0} e^{i k z+\alpha t} & r \geq a
\end{array}
$$

where $K(k r)$ is the modified Bessel function of the second kind and the normal stress in gas is obtained from $\pi_{G, r r}=p_{G}$.

Substituting the values found for $\pi_{r r}, \pi_{G, r r}$ and $p_{\sigma}$ into the boundary condition of Equation (21) leads to the following dispersion relation for an axisymmetric non-Newtonian viscoelastic liquid jet: 


$$
\begin{gathered}
-\frac{\rho(\alpha+i k \bar{U})^{2}\left(l^{2}+k^{2}\right) \eta(\alpha) \xi_{0}}{k\left(l^{2}-k^{2}\right)} \frac{I_{0}(k r)}{I_{1}(k a)}+\frac{\rho_{G} \xi_{0}\left(\alpha+i k \bar{U}_{G}\right)^{2}}{k} \frac{K_{0}(k r)}{K_{0}^{\prime}(k a)}+\sigma\left(\frac{1}{a^{2}}-k^{2}\right) \xi_{0}+ \\
2 \eta(\omega)\left[\frac{\left(l^{2}+k^{2}\right)\left(I_{0}(k a)-\frac{1}{k a} I_{1}(k a)\right)}{I_{1}(k a)}-\frac{2 k^{2} l\left(I_{0}(l a)-\frac{1}{l a} I_{1}(l a)\right)}{I_{1}(l a)}\right] \frac{\eta(\omega) \xi_{0}}{\rho}=0
\end{gathered}
$$

Equation (46) relates the complex frequency of a disturbance to its real wave number $\mathrm{k}$. In order to solve the equation for $\omega$, keep in mind that the parameter $l$ is a function of $\omega$.

It is convenient that the dispersion relation is expressed in non-dimensional form. After non-dimensionalizing and rearranging, we get the final dispersion equation as Equation (47). On solving for variable dimensionless wave number $\mathrm{k}$, we get the real part of the solution as dimensionless growth rate $\varpi$ for a set of values of other dimensionless parameters such as We, $\mathrm{El}, \mathrm{Z}, \bar{\rho}$ and $\bar{\lambda}$.

$$
\begin{gathered}
-\frac{(\bar{\omega}+i \bar{k} \sqrt{W e}) I_{0}(\bar{k})}{\bar{k} I_{1}(\bar{k})}\left[\frac{2 \bar{k} z(z+\bar{\lambda} \bar{\omega} E l)}{(z+\bar{\omega} E l)}+(\bar{\omega}+i \bar{k} \sqrt{W e})\right]-\frac{\bar{\rho}}{\bar{k}}\left(\bar{\omega}+i \bar{k} U_{r} \sqrt{W e}\right)^{2} \frac{K_{1}(\bar{k})}{K_{0}(\bar{k})+\frac{1}{\bar{k}} K_{1}(\bar{k})}+ \\
\left(1-\bar{k}^{2}\right)-\frac{2 z^{2}(z+\bar{\lambda} \bar{\omega} E l)^{2}}{(z+\bar{\omega} E l)^{2}}\left[\begin{array}{c}
\left(2 \bar{k}^{2}+\frac{(\bar{\omega}+i \bar{k} \sqrt{W e})(z+\bar{\omega} E l)}{z(z+\bar{\lambda} \bar{\omega} E l)}\right)\left(\frac{I_{0}(\bar{k})}{I_{1}(\bar{k})}-\frac{1}{k}\right)- \\
2 k^{2}\left(\frac{I_{0}(l a)}{I_{1}(l a)}-\frac{1}{\bar{k}^{2}+\frac{(\bar{\omega}+i \bar{k} \sqrt{W e})(z+\bar{\omega} E l)}{z(z+\bar{\lambda} \bar{\omega} E l)}}\right)
\end{array}\right]=0
\end{gathered}
$$

where $K=k a$ is the dimensionless wave number and $W e=\frac{\rho U^{2} a}{\gamma}$ is the liquid Weber number, which expresses the ratio of inertia forces to tension surface forces. The Ohnesorge number $z=\frac{\eta_{0}}{\sqrt{(\rho \gamma a)}}$ denotes the ratio of viscous forces to surface tension forces. The elasticity number $E l=\frac{\lambda_{1} \eta_{0}}{\left(\rho a^{2}\right)}$ is used to describe the relationship between viscous and elastic effects in the liquid jet. In Equation (47), $\bar{\rho}=\frac{\rho_{G}}{\rho}$ is the ratio of gas-to-liquid density, $\bar{\lambda}=\frac{\lambda_{2}}{\lambda_{1}}$ is the ratio of deformation retardation time to stress relaxation time, $U_{r}=\frac{U_{G}}{U}$ is the ratio of gas-to-liquid axial velocity, and $\bar{\omega}=\frac{\omega}{\sqrt{\frac{\sigma}{\rho a^{3}}}}$ is the dimensionless growth rate. When $\lambda_{1}=\lambda_{2}=$ 0 , the jet of a viscoelastic fluid is transformed into that of Newtonian fluid. At this condition, $\eta_{0}=\mu$, where $\mu$, is the dynamic viscosity of Newtonian fluid. The Newtonian fluid distribution equation is obtained by rewriting Eqution (47) with the mentioned assumptions:

$$
\begin{array}{r}
-\frac{(\bar{\omega}+i \bar{k} \sqrt{W e}) I_{0}(\bar{k})}{\bar{k} I_{1}(\bar{k})}[2 \bar{k} z+(\bar{\omega}+i \bar{k} \sqrt{W e})]-\frac{\bar{\rho}}{\bar{k}}\left(\bar{\omega}+i \bar{k} U_{r} \sqrt{W e}\right)^{2} \frac{K_{1}(\bar{k})}{K_{0}(\bar{k})+\frac{1}{\bar{k}} K_{1}(\bar{k})}+ \\
\left(1-\bar{k}^{2}\right)-2 z^{2}\left[\left(2 \bar{k}^{2}+\frac{(\bar{\omega}+i \bar{k} \sqrt{W e})}{z}\right)\left(\frac{I_{0}(\bar{k})}{I_{1}(\bar{k})}-\frac{1}{k}\right)-2 k^{2}\left(\frac{I_{0}(l a)}{I_{1}(l a)}-\frac{1}{\bar{k}^{2}+\frac{(\bar{\omega}+i \bar{k} \sqrt{W e})}{z}}\right)\right]=0
\end{array}
$$

when the liquid viscosity vanishes, the jet of the Newtonian fluid is transformed into that of an inviscid fluid, and the dispersion Equation (48) reduces to the results for an inviscid fluid: 


$$
-\frac{(\bar{\omega}+i \bar{k} \sqrt{W e}) I_{0}(\bar{k})}{\bar{k} I_{1}(\bar{k})}(\bar{\omega}+i \bar{k} \sqrt{W e})-\frac{\bar{\rho}}{\bar{k}}\left(\bar{\omega}+i \bar{k} U_{r} \sqrt{W e}\right)^{2} \frac{K_{1}(\bar{k})}{K_{0}(\bar{k})+\frac{1}{\bar{k}} K_{1}(\bar{k})}+\left(1-\bar{k}^{2}\right)=0
$$

\section{PRIMARY BREAKUP THEORY}

In order to prove the theory of this paper, non-dimensional breakup lengths were calculated to investigate the effects of each of the liquid properties on the breakup length. According to Brenn et al. [20], the non-dimensional breakup length $\frac{L_{b}}{2 a}$ can be calculated using the dimensional maximum growth rates of the disturbances in the Equation (50).

$$
\frac{L_{b}}{2 a}=c_{1} \frac{U}{2 a \omega_{\max }}
$$

In the above equation, $\mathrm{U}$ is the absolute velocity of the liquid, $L_{b}$ is the primary breakup length of the liquid jet, and $c_{1}$ is an empirical coefficient. Brenn et al. (20) indicate that based on the fit of the experiment results, this coefficient is set to be 3.6.

\section{RESULT}

The dimensionless growth rates were obtained by solving the dispersion equation (Equation (47)) using Muller's Secant method, which searches for a numerical solution to a given equation with initialized values for the independent variable. The positive real part of the complex number of wave frequency represents the degree of the jet instability.

\subsection{Comparison with other theories}

In order to validate the present model, the results were obtained for the cases considered by Brenn et al. [20] for non-Newtonian viscoelastic liquid jet instability in stagnant air. The dimensional growth rates were calculated as a function of dimensionless wave number for,

$$
\begin{gathered}
U_{r}=0 \frac{\mathrm{m}}{\mathrm{s}}, a=0.921 \mathrm{~mm}, \rho=1000 \frac{\mathrm{kg}}{\mathrm{m}^{3}}, \rho_{G}=1.2 \frac{\mathrm{kg}}{\mathrm{m}^{3}}, \sigma=0.07 \frac{\mathrm{N}}{\mathrm{m}}, \eta_{0}=0.11 \text { Pa.s } \\
\lambda_{1}=0.1 \mathrm{~ms}, \lambda_{2}=0.01 \mathrm{~ms} .
\end{gathered}
$$

From Figure 2, it can be seen that the results match well with the results from Brenn et al. (20).

Figure 3 shows the comparison of dimensionless growth rate $\bar{\omega}$ as a function of the dimensionless wave number $\mathrm{K}$ for inviscid, non-Newtonian and Newtonian liquid jet in stagnant air under axisymmetric disturbances. The parameters are fixed at $\bar{\rho}=0.001, \bar{U}=0$, $\bar{\lambda}=0.1, E l=1, z=0.5, W e_{l}=100$. It can be seen from Figure 3 that the growth rate of disturbances for non-Newtonian fluid is more significant than that of the Newtonian fluid and smaller than that of an inviscid fluid. The inviscid liquid jet is more unstable than the viscoelastic liquid, which again has a higher growth rate or instability compared to the viscous Newtonian liquid. This can be explained by the elastic nature of these two types of viscous liquids. In a viscoelastic jet, the elastic freedom is higher; consequently, the Newtonian jet appears more stable or rigid, and the viscoelastic jet is more prone to deformation. Similar results were reported by Liu et al. (21) in their studies of the axisymmetric instability of nonNewtonian jets. Because of additional elastic freedom for deformation, a non-Newtonian liquid jet is more unstable than a Newtonian liquid jet. 


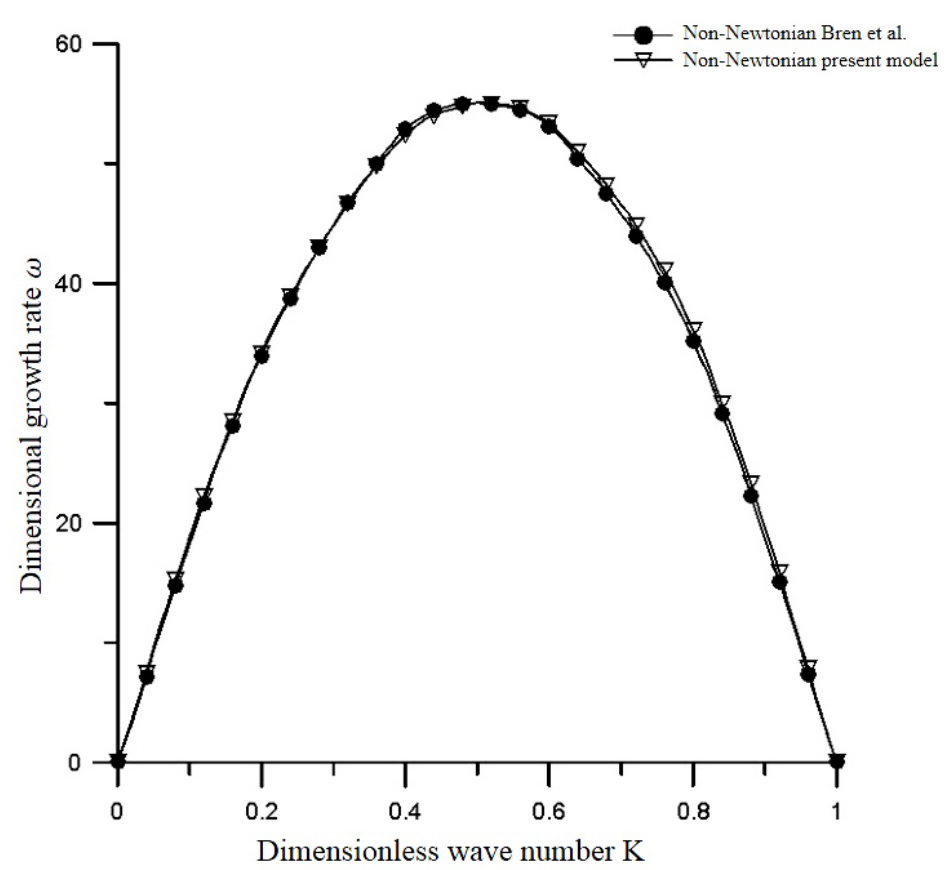

Figure 2: Variation of Dimensional Growth Rate with Dimensionless Wave Number in stagnant gas surrounding using data of Brenn et al. [20].

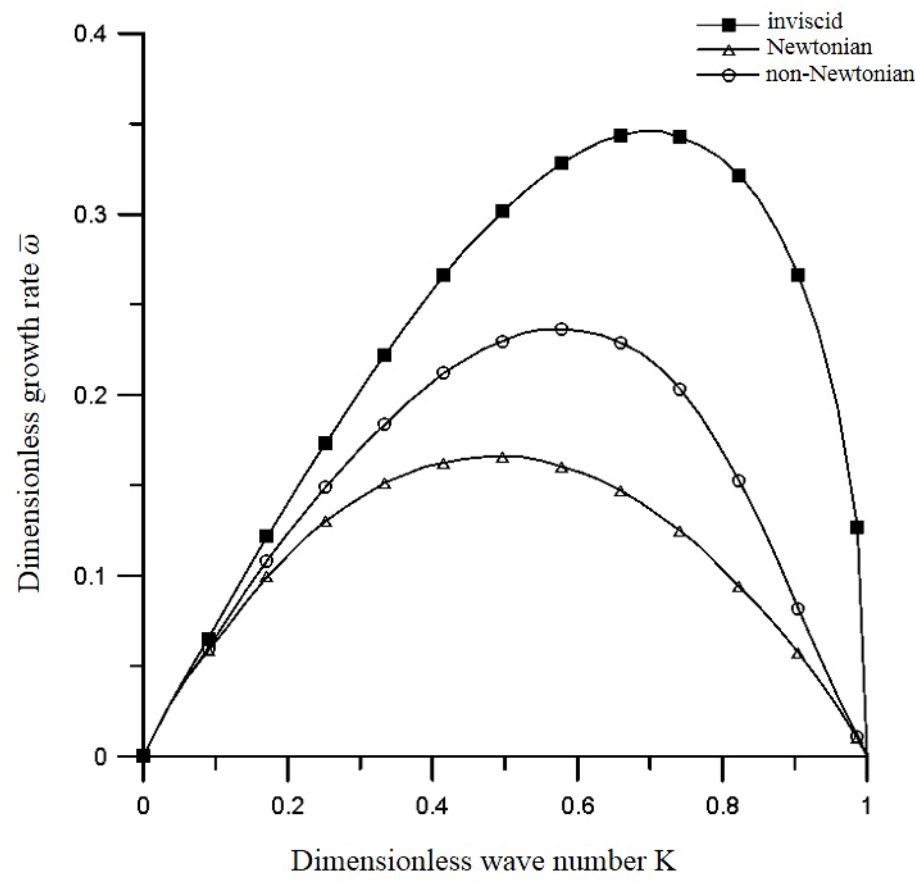

Figure 3: Dimensionless Wave Number versus Dimensionless Growth Rate for different liquid jets 
In Figure 4, the non-dimensional breakup length $\frac{L_{b}}{2 a}$ is shown as a function of the Weber number for non-Newtonian, Newtonian and inviscid liquid jets. The inspection of this figure shows that the non-dimensional breakup length for Newtonian fluid was more significant than that for non-Newtonian and inviscid fluids with the same Weber number; therefore, it can be concluded that elasticity exhibits a destabilization effect in the breakup process of nonNewtonian jets. While Newtonian jets appear rigid, non-Newtonian jets have the additional freedom for elastic deformation. Other authors have also reported similar results [20-22].

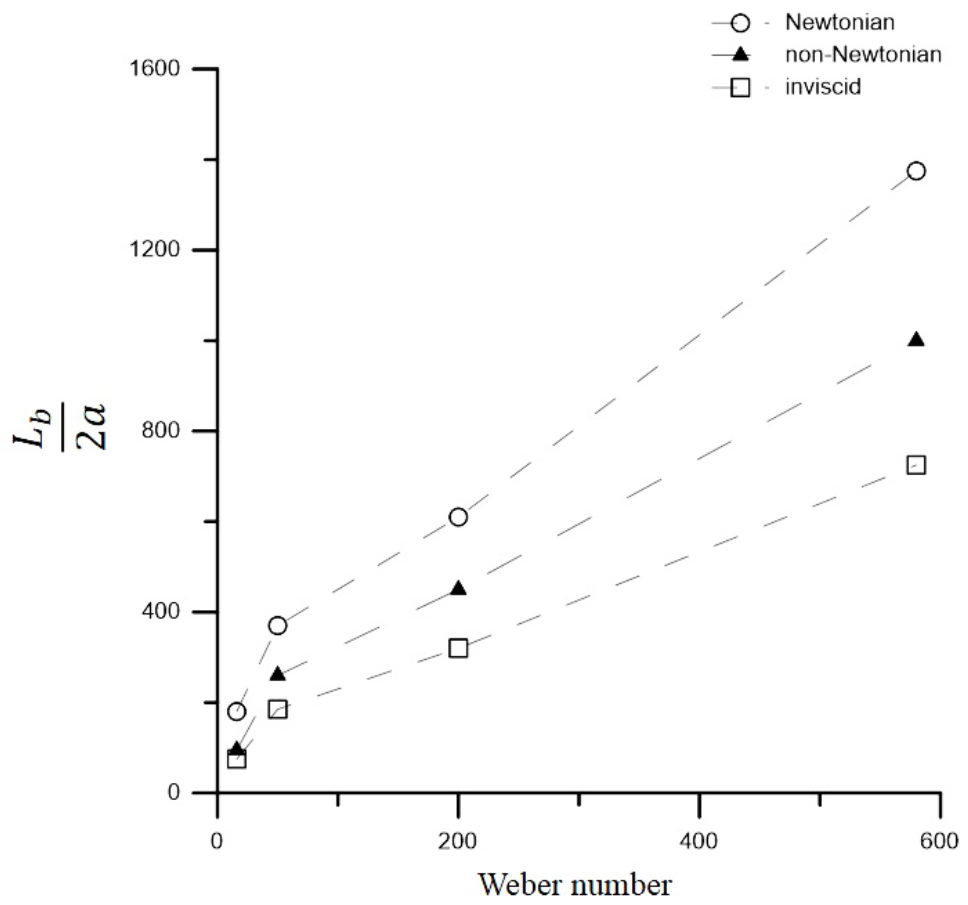

Figure 4: Effects of Weber number on non-dimensional breakup length for different liquid jets

\subsection{Effects of fluid properties}

The breakup mechanism from a jet into drops, the breakup length, and the resultant drop diameter are influenced by the fluid properties, primarily by the viscoelastic nature of the liquid. The effects of various fluid properties in non-dimensional forms such as the Weber number, ratio of liquid to gas densities, and time constant ratio on the growth rate and breakup of non-Newtonian liquid jets are studied.

\subsubsection{Effects of elasticity and time constant ratio}

The elasticity number (El) is directly proportional to the elastic force and inversely proportional to the inertial force. Figure 5 show the results for $\mathrm{We}=30, \mathrm{Z}=0.5, \bar{\lambda}=0.1$ and the elasticity number is varied from 0.5 to 5 . It is observed that increasing the elasticity number 
raises the disturbance growth rate. The liquid elasticity characteristic always tends to increase the wave growth rate in non-Newtonian viscoelastic liquid jets for axisymmetric disturbances. Thus, it could be said that non-Newtonian fluids are more unstable than their Newtonian counterparts because of $E l=0$ for Newtonian fluids.

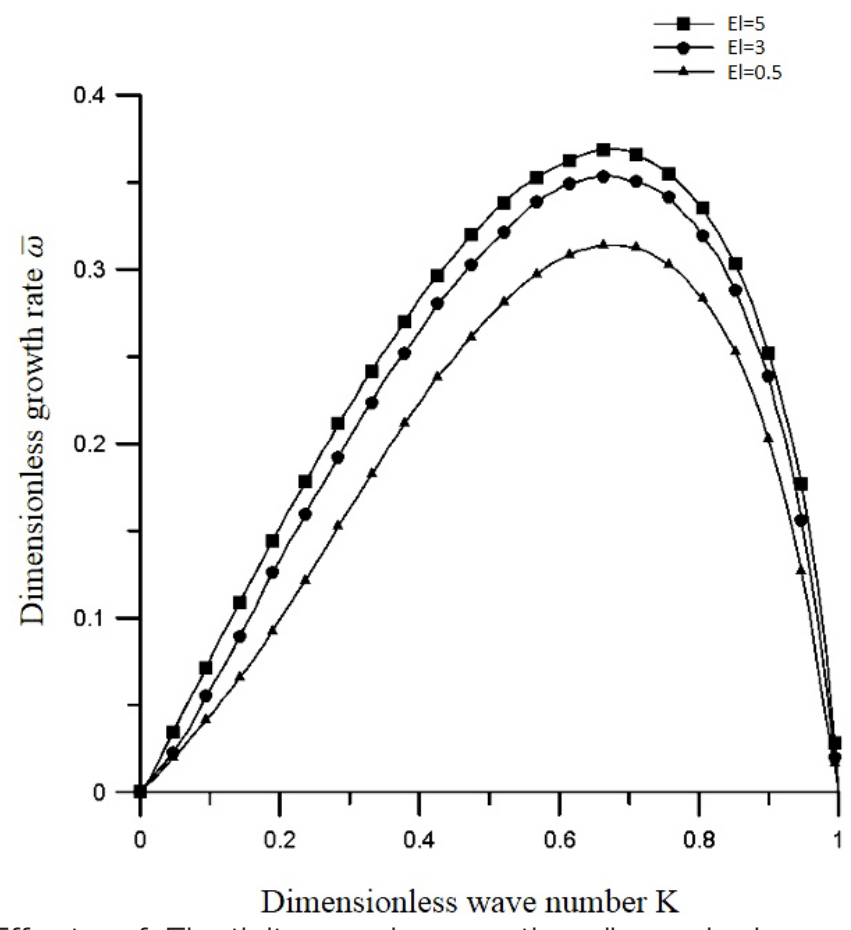

Figure 5: Effects of Elasticity number on the dimensionless growth rate $\bar{\omega}$ of disturbance on different non-Newtonian viscoelastic liquid jets $v s$. the dimensionless wave number $\mathrm{K}$

The time constant ratio $(\bar{\lambda})$ is the ratio of deformation retardation time to the stress relaxation time. The time constant ratio can be changed by either changing the deformation retardation time $\left(\lambda_{2}\right)$ or the stress relaxation time $\left(\lambda_{1}\right)$ or both. Changing the retardation time $\left(\lambda_{2}\right)$ changes the time constant ratio alone and keeps the elasticity number constant, while any change in the relaxation time $\left(\lambda_{1}\right)$ changes both the time constant ratio and elasticity number. Therefore, the relaxation time is held constant, while the retardation time increases. To see the effects of time constant ratio on the growth rate of non-Newtonian viscoelastic liquid jets instabilities, the time constant ratio is varied from 0.1 to 0.7 while other parameters were kept at $\mathrm{We}=30, \mathrm{Z}=0.5$, and $E l=4$. The results are shown in Figure 7 . It is evident that as the time constant ratio is reduced, the growth rates of disturbances increase correspondingly. Because of the increasing stress tensor of the viscoelastic liquid jet due to increasing the deformation retardation time, the liquid jet dissipates more energy and will become more stable. Also, with the varying time constant ratio, the instability range remains the same.

An increase in the time constant ratio results in increasing the breakup length of the liquid jet, irrespective of a change in the elasticity number. The effects of the time constant ratio on the non-dimensional breakup length of the viscoelastic liquid jet with the same parameters as mentioned above are shown in Figure 8. 


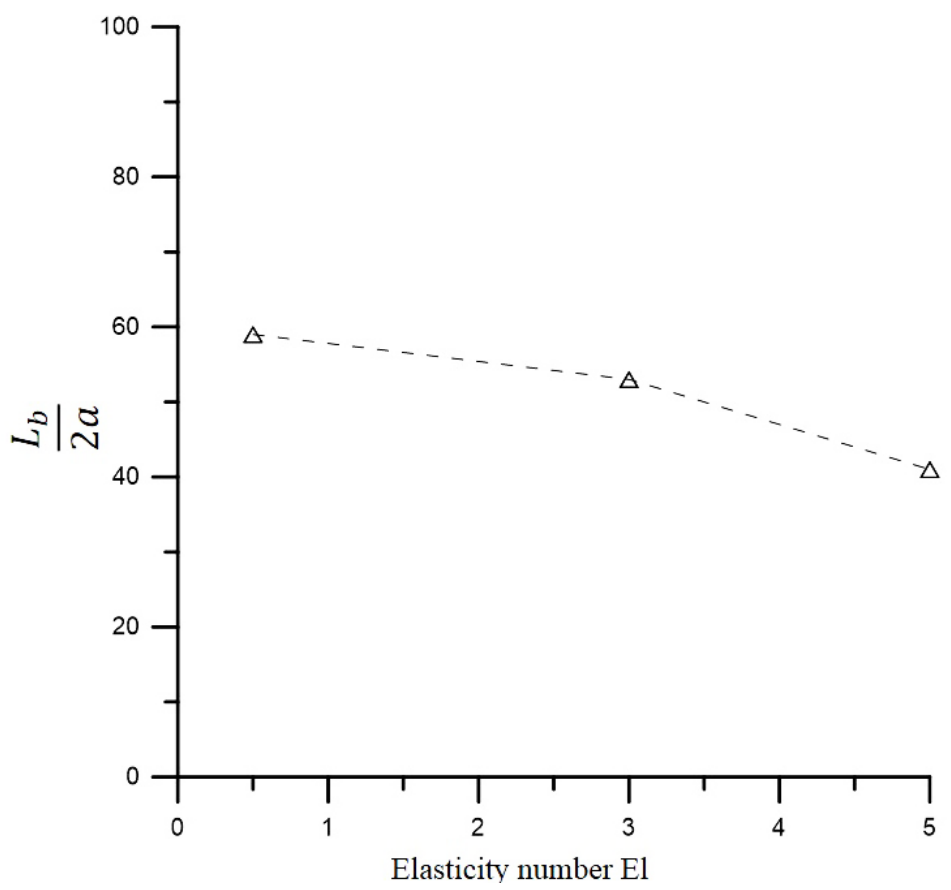

Figure 6: Effects of Elasticity number on the dimensionless breakup length

To see the effects of the time constant ratio on the breakup of the liquid jet, two different cases are assumed as tabulated in Table 1. In the first case, the relaxation time is held constant, while the retardation time is increased. It can be seen from Table 1 that by increasing the retardation time $\left(\lambda_{2}\right)$, the elasticity number remains constant. However, there is an apparent increase in the breakup length of the liquid jet. Also, it can be inferred that there is a decrease in almost all of the wave numbers as the time constant ratio increases. In the second case, retardation time is held constant, while the relaxation time is increased. It can be seen from Table 1 that by increasing the relaxation time $\left(\lambda_{1}\right)$ the elasticity number decreases. However, there is a clear increase in the breakup length of the liquid jet; there is no change in the most dominant wave number as the time constant ratio increases. It can be inferred that the time constant ratio has a significant effect on determining the breakup length of the jet. With an increase in the time constant rate, the breakup length of the liquid jet is stretched out. This has also been reported by many researchers $[15,19]$.

Table 1: Effect of deformation retardation time and stress relaxation time on breakup length

\begin{tabular}{ccccc|cccc}
\hline \multicolumn{5}{c|}{ Case 1 } & \multicolumn{5}{c}{ Case 2 } \\
\hline $\bar{\lambda}$ & 0.1 & 0.3 & 0.5 & 0.7 & 0.083 & 0.0625 & 0.05 & 0.033 \\
$\lambda_{\mathbf{1}}(\mathrm{ms})$ & 10 & 10 & 10 & 10 & 12 & 16 & 20 & 30 \\
$\boldsymbol{\lambda}_{\mathbf{2}}(\mathrm{ms})$ & 1 & 3 & 5 & 7 & 1 & 1 & 1 & 1 \\
$\mathrm{El}$ & 4 & 4 & 4 & 4 & 0.77 & 1.3 & 1.52 & 2.75 \\
$\frac{\boldsymbol{L}_{\boldsymbol{b}}}{\mathbf{2 a}}$ & 31.2 & 38.7 & 42.3 & 49.1 & 41.6 & 39.8 & 37.2 & 35.4 \\
\hline
\end{tabular}




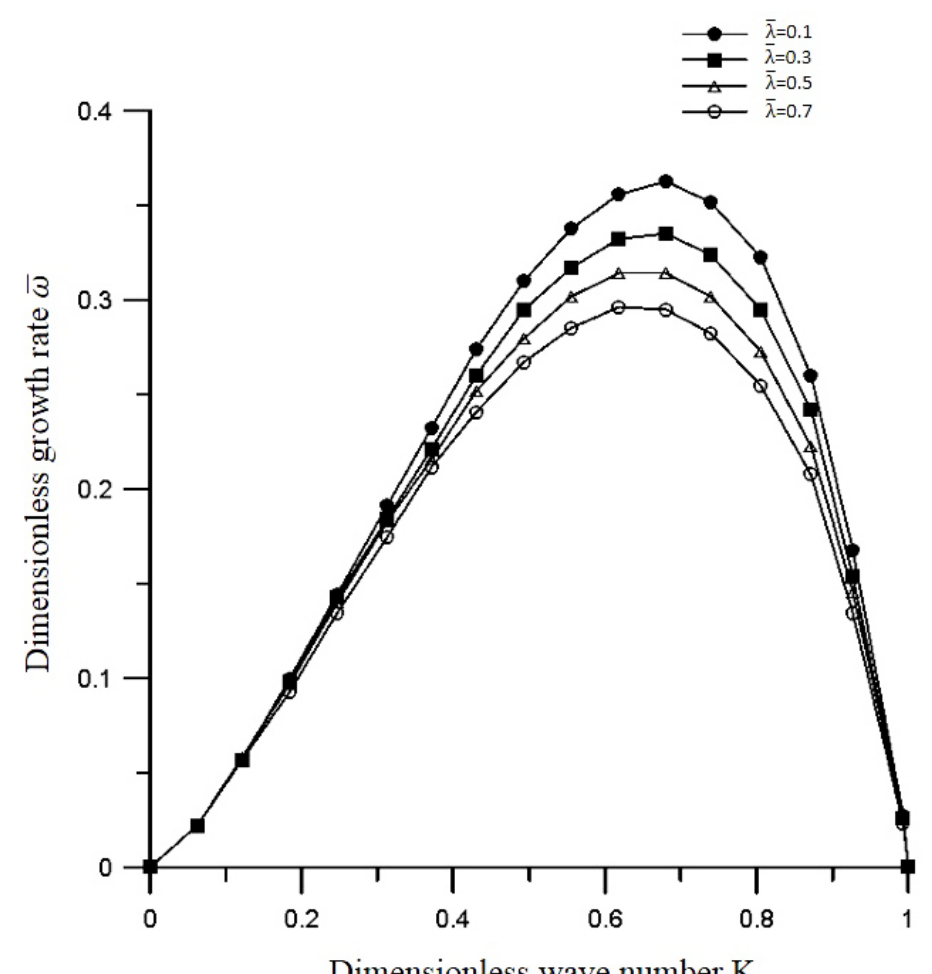

Dimensionless wave number $\mathrm{K}$

Figure 7: Effects of time constant ratio on the dimensionless growth rate $\bar{\omega}$ of disturbance on different non-Newtonian viscoelastic liquid jet $v s$. the dimensionless wave number $\mathrm{K}$

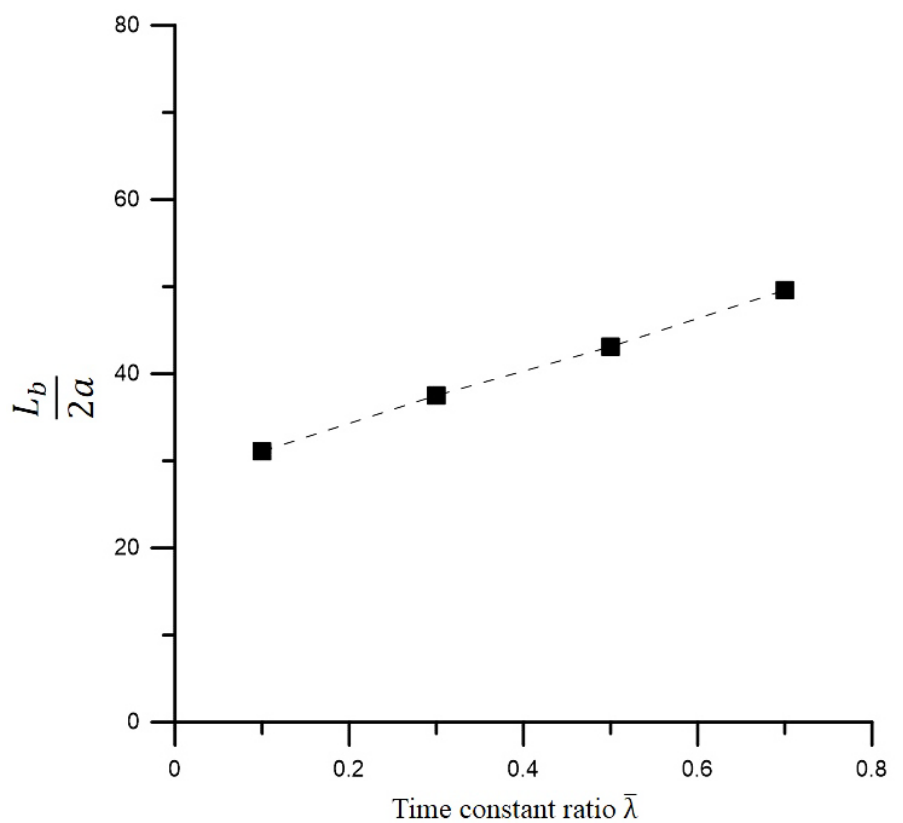

Figure 8: Effects of time constant ratio on the dimensionless breakup length 


\subsubsection{Effects of Weber number}

The Weber number defines the relation between the inertial force and the surface tension force of the fluids. An increase of the Weber number may be caused either by a rise in the jet velocity, radius or density or by decreasing surface tension. In fluid dynamics for this case, if the surface tension, jet radius, and density are constant, then this number signifies the effects of jet velocity on the fluid. The aerodynamic interaction between the liquid and gas medium that cause the instability of liquid jet can be increased by increasing the jet velocity. On the other hand, the surface tension resists the onset and development of short-wave instability on non-Newtonian viscoelastic liquid jets. It smoothens the disturbances on the interface between the liquid and the gas.

It can be shown that a jet with a higher Weber number is more unstable than the same jet at a lower Weber number. The Weber number affects the growth rate of unstable waves directly so that the most unstable wave number and the instability range become widespread. The reason for this behavior is attributed to the velocity and the surface tension of the liquid jet. The surface tension stabilizes the liquid jet; it inhibits the onset and growth of the disturbance. At a lower Weber number, i.e., when the velocity of the jet is low, the surface tension tends to play the more significant role. On the other hand, at a higher Weber number, the disturbances freely overcome the surface tension and makes the jet unstable. This result indicates that by increasing the jet velocity, the stability of the non-Newtonian viscoelastic liquid jet is reduced as shown in Figure 9. As the disturbance grows, the unstable wave amplitude becomes higher and thus leads to the breakup of the liquid jet.

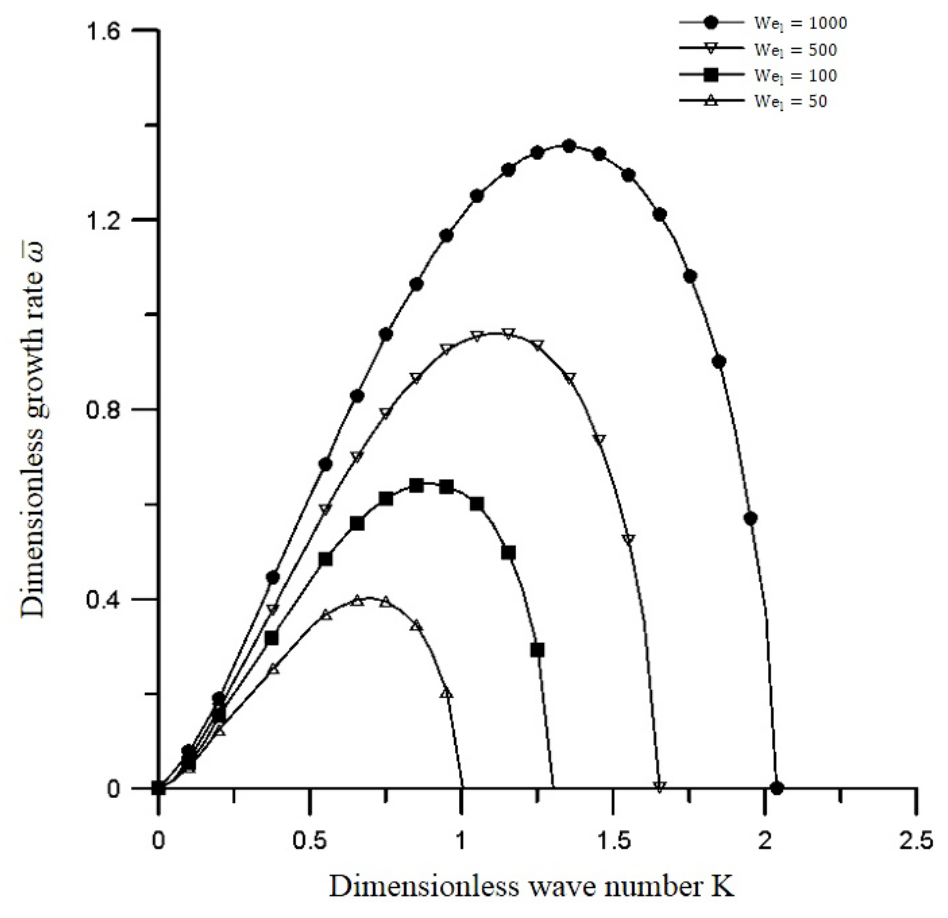

Figure 9: Effects of Weber number on the dimensionless growth rate $\bar{\omega}$ of disturbance on different non-Newtonian viscoelastic liquid jets vs. the dimensionless wave number $\mathrm{K}$ 
Figure 10 shows the effect of the Weber number on the resultant breakup length for all parameters that were fixed the same as mentioned earlier. It is seen that as the Weber number increased, the breakup length of the viscoelastic jet increased which has been reported by other authors. Increasing the velocity of the liquid jet resulted in reducing the amplitude of unstable waves at the near-nozzle region. The breakup length growth showed that the aerodynamic interaction with the liquid jet column was dominant at the far from the near-nozzle area.

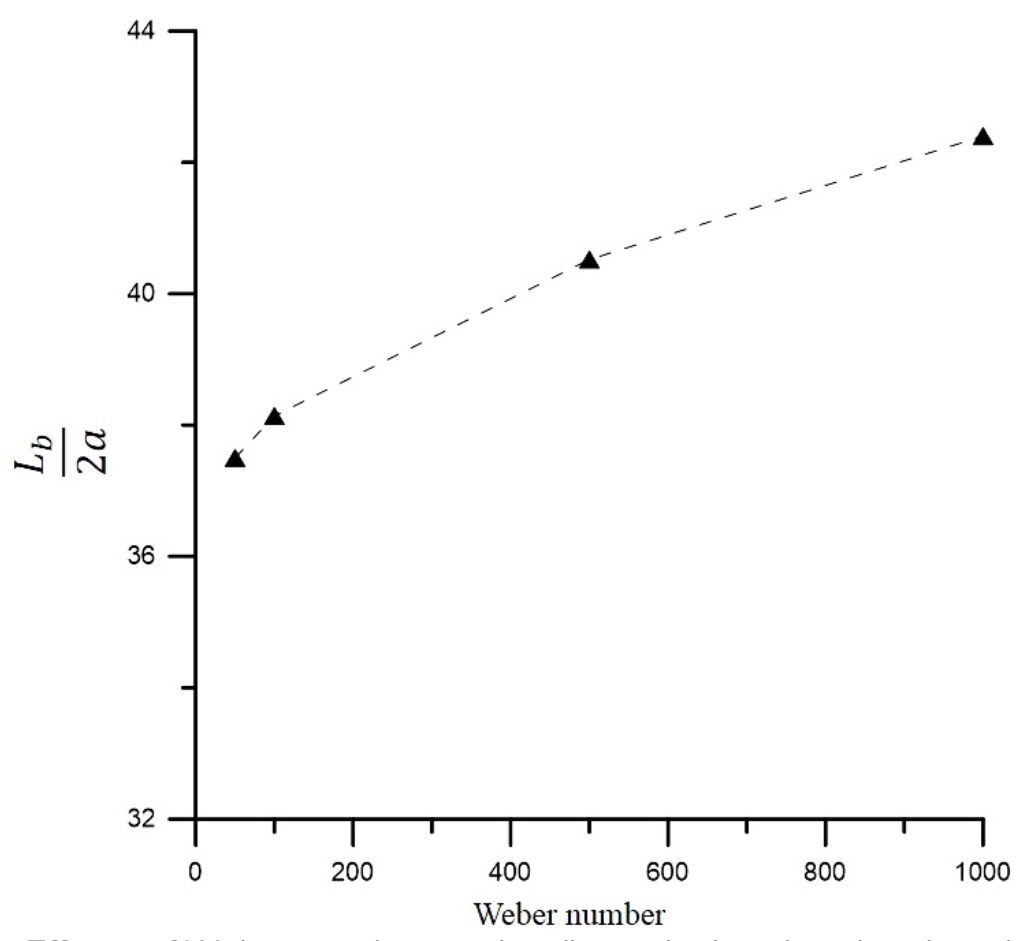

Figure 10: Effects of Weber number on the dimensionless breakup length

\subsubsection{Effects of Ohnesorge number}

The Ohnesorge number $(Z)$ is a dimensionless number that relates the viscous force to the inertial and surface tension forces. It can be expressed as the ratio of the square root of the Weber number over the Reynolds number. If the velocity and surface tension of the jet is held constant for any given liquid jet, then any change in this number is attributed to the effects of viscosity alone. Thus, changing the Ohnesorge number reflects a change in the inherent fluid viscosity. Hence, the higher Ohnesorge number corresponds to higher viscosity. In the physical sense, if the viscosity of the liquid undergoing atomization increases, then one would expect a higher breakup length compared to a liquid of lesser viscosity because the time taken for the disturbance to propagate is higher for the same Weber number.

Figure 11 is obtained for $W e=30, E l=4.4, \bar{\lambda}=0.1$ and the Ohnesorge number varies from 0.5 to 2 . From Figure 11 we can deduce that as the Ohnesorge number increases from 0.5 to 2 , the jet becomes more and more stable. Furthermore, a rise in the Ohnesorge number could decrease the growth rate as well as the most dominant wave number. While velocity acts as an aiding force by increasing the disturbance growth rate, the liquid viscosity acts as a 
restraining force by increasing the stability of the liquid jet. The viscosity dampens the growth of the surface disturbances thus preventing it from breaking up. Also, it can be concluded from Figure 11 that even though there is a change in the most dominant wave number, the instability range does not change with the Ohnesorge number.

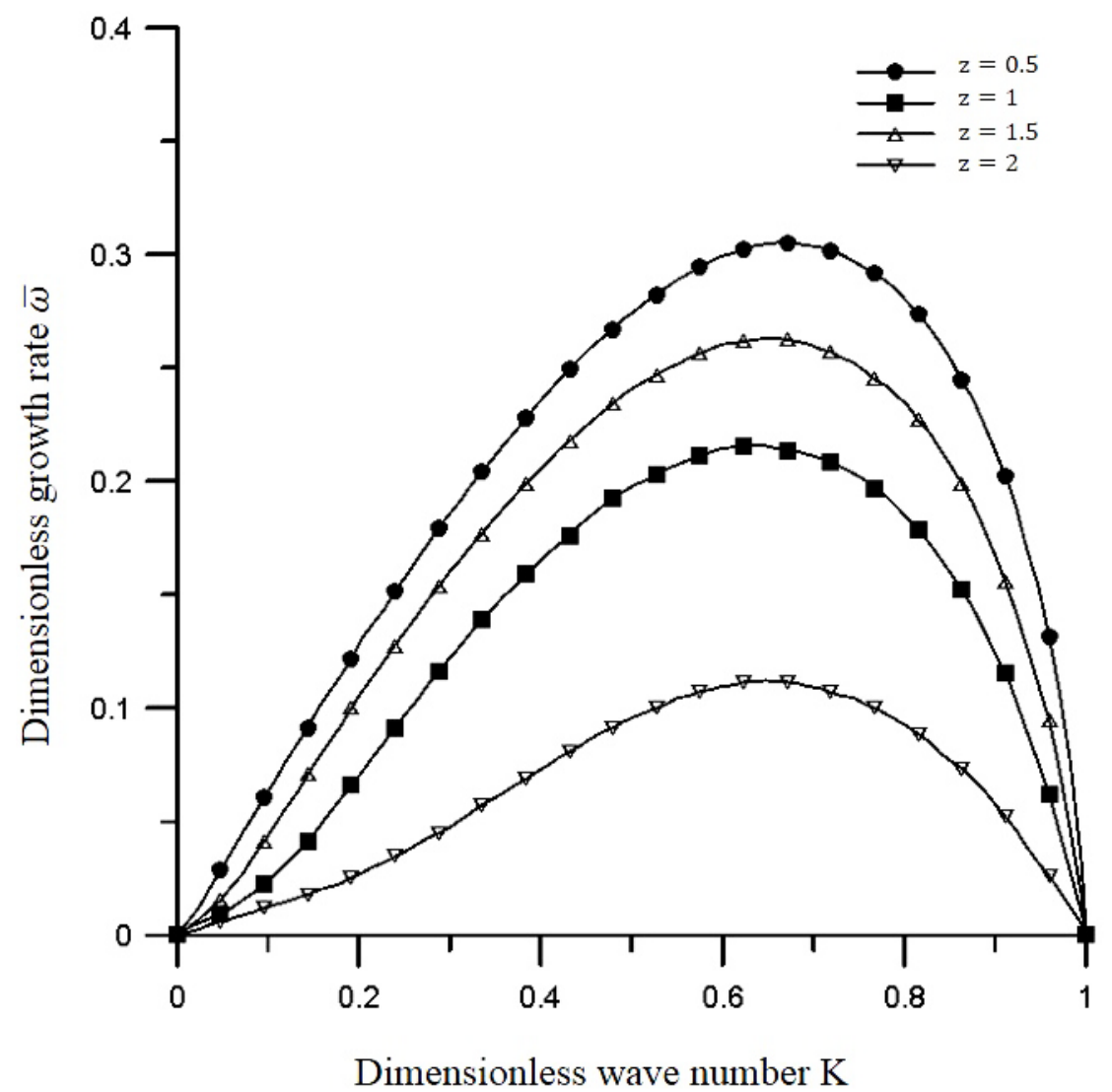

Figure 11: Effects of Ohnesorge number on the dimensionless growth rate $\bar{\omega}$ of disturbance on different non-Newtonian viscoelastic liquid jets vs. the dimensionless wave number $\mathrm{K}$

Figure 12 shows the effect of the Ohnesorge number, which is primarily due to liquid viscosity, on the resultant non-dimensional breakup length. The breakup length for viscoelastic liquid jet increases as the Ohnesorge number increases, which is a clear indication that the viscosity dampens the growth of the disturbance. From Figure 12, we can also conclude that as the viscosity increases, the length of the breakup increases. Thus, the viscosity of the liquid plays a vital role in the dynamics of the liquid jet breakup. 


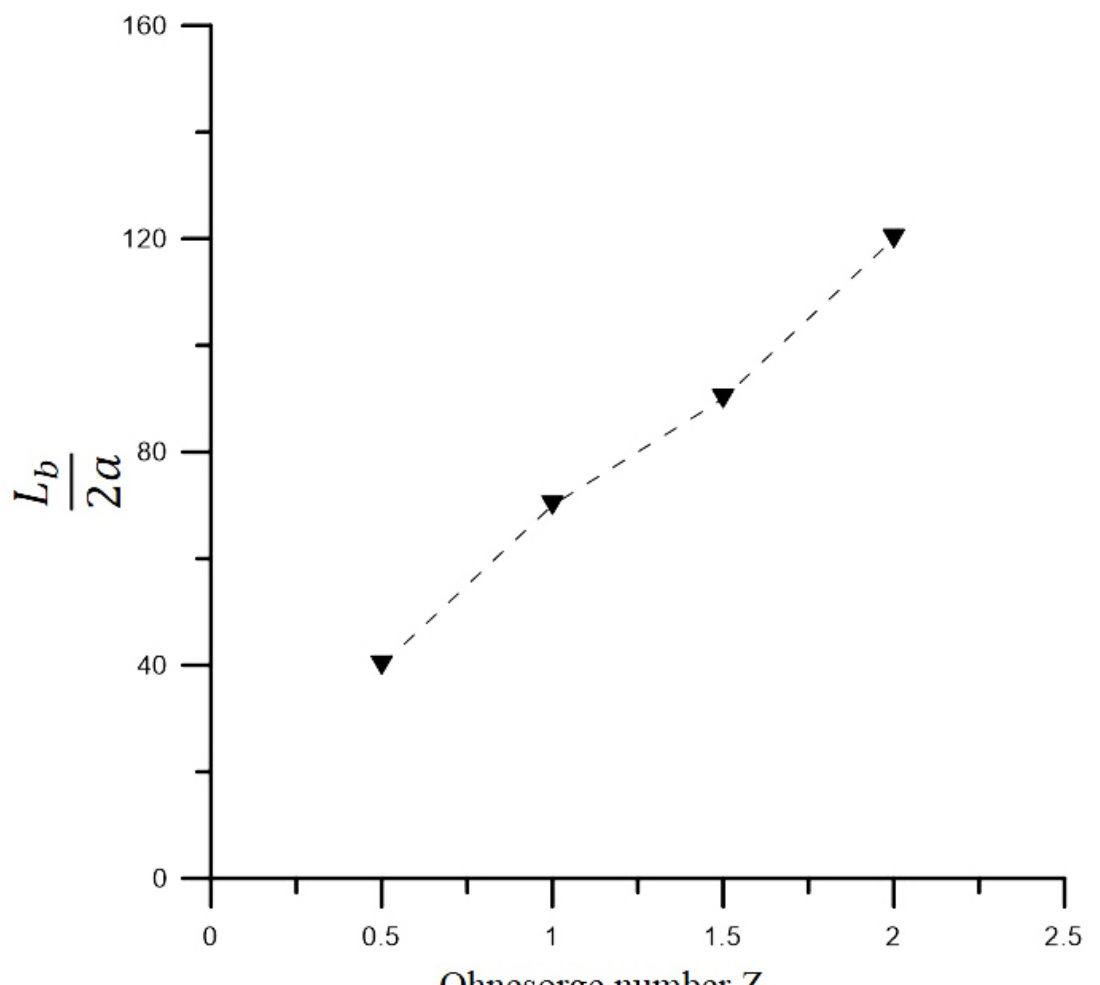

Ohnesorge number $\mathrm{Z}$

Figure 12: Effects of Onnesorge number on the dimensionless breakup length

\subsubsection{Effects of the gas-to-liquid density ratio}

The effects of the gas-to-liquid density ratio on the disturbance growth rates are shown in Figure 13 at $W e=500, E l=4, z=0.5$ and $\bar{\lambda}=0.1$. Figure 13 indicates the effect of ambient gas to liquid density ratio on disturbance growth rates. Increasing the gas-to-liquid density ratio leads to increased growth rates, which means the gas to liquid density ratio can accelerate the disturbance. The aerodynamic force of the surrounding gas is related to gas density. By increasing the gas density, the aerodynamic interaction that exist at the interface of the liquid jet will become stronger and lead to more instability of the viscoelastic liquid jet. Therefore, the ambient gas with higher density could result in the enhancement of the non-Newtonian viscoelastic liquid jet instability. It should be noted that gas density can be improved by increasing the gas pressure. Thus, a high gas pressure may be beneficial for jet instability.

Figure 14 shows the effect of the gas-to-liquid density ratio on the non-dimensional breakup length by varying the gas density from $1 \mathrm{~kg} / \mathrm{m} 3$ to $5 \mathrm{~kg} / \mathrm{m} 3$ and keeping the other parameters constant. It can be seen from Figure 14 that by increasing the gas-to-liquid density ratio, the non-dimensional breakup length of the viscoelastic liquid jet decreases. It is found that increasing gas density can accelerate the breakup of a viscoelastic liquid jet to an equal degree in all the reachable ranges. 


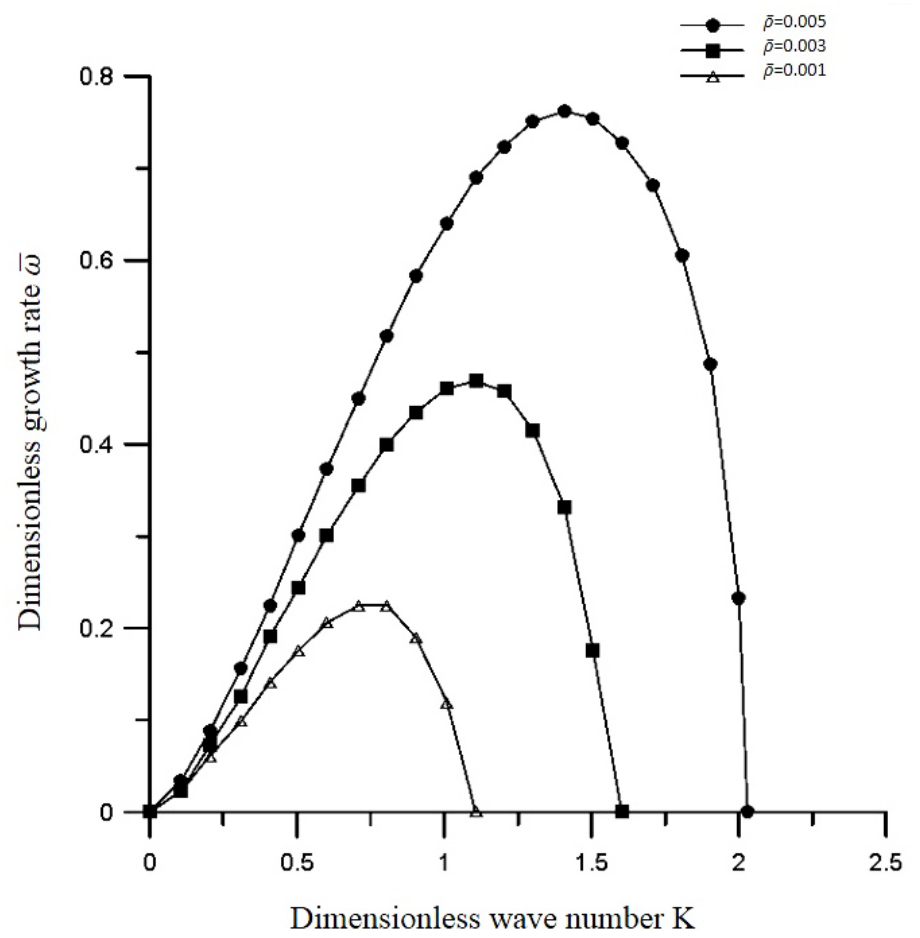

Figure 13: Effects of the gas-to-liquid density ratio on the dimensionless growth rate $\bar{\omega}$ of disturbance on different non-Newtonian viscoelastic liquid jets vs. the dimensionless wave number $\mathrm{K}$

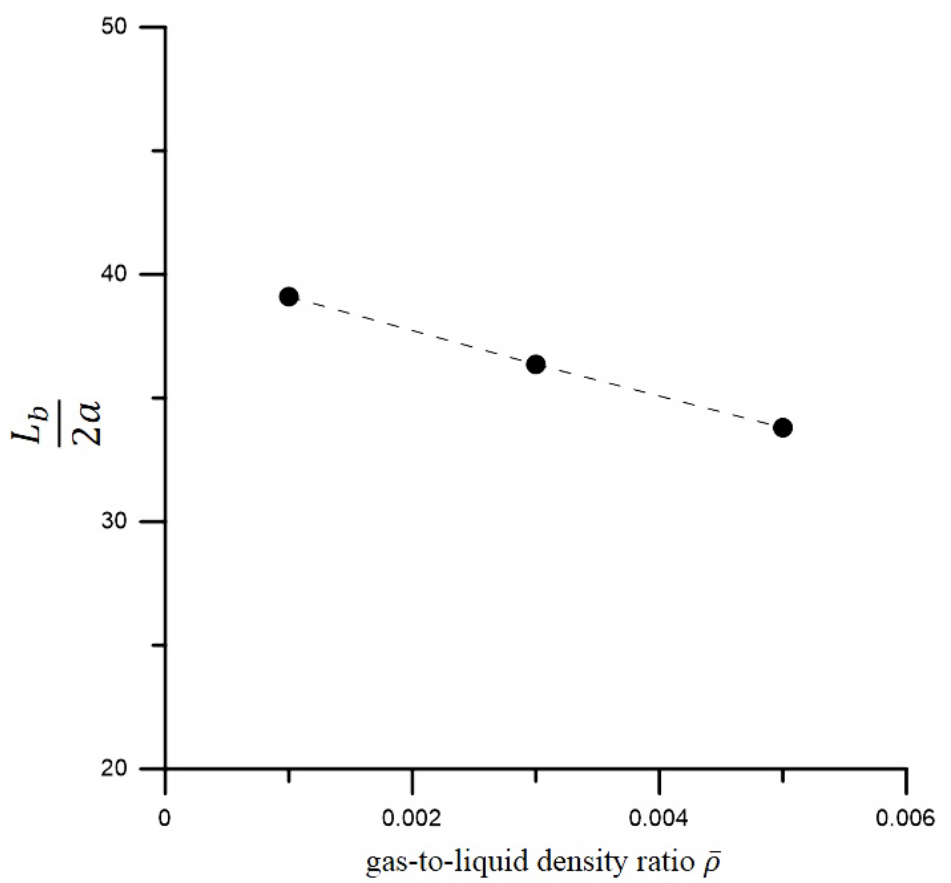

Figure 14: Effects of the gas-to-liquid density ratio on the dimensionless breakup length 


\section{CONCLUSION}

The instability and breakup of non-Newtonian viscoelastic liquid jets with axisymmetric disturbances moving in an inviscid gaseous environment was investigated in this article. A dispersion relation for axisymmetric disturbances on a non-Newtonian viscoelastic liquid jet was derived from linearized governing equations and the constitutive equation of the non-Newtonian fluid. Moreover, the dispersion relation of Newtonian and inviscid liquid jet was obtained. The model developed was validated by comparing the results with other theoretical models available in the literature. The results showed that inviscid liquid jet was more unstable than the viscoelastic liquid which again had a higher growth rate or instability compared to the viscous Newtonian liquid jets. This phenomenon can be explained by the elastic behaviors of the two types of viscous liquids. The elastic freedom in a viscoelastic jet was more than the others. Hence, the Newtonian jet appeared more stable or rigid, and the viscoelastic jet was more prone to deformation. This result has been obtained by other authors. To prove the theory of this paper, the non-dimensional breakup lengths of the viscoelastic liquid jet were calculated, and the effects of fluid properties on the breakup of the liquid jet were investigated.

The results showed that increasing the elasticity number and decreasing the time constant ratio $\bar{\lambda}$ led to increased maximum growth rates of axisymmetrical disturbances on the jets and therefore, decreased the non-dimensional breakup length. Increasing the relative velocity between the jet and the ambient gas was the source of increased growth rates of instabilities. Increasing the Weber number of the liquid jet resulted in the enhancement of the disturbances growth rate. Similar results were previously obtained for inviscid and Newtonian liquid jets by many other authors. An increase of the ratio of gas-to-liquid density or the Weber number enhanced both the growth rate and the instability range of the non-Newtonian viscoelastic liquid jets for the conditions investigated here. The results indicated that a high-density environment led to an enhancement of the instability and lower breakup length of the jet. The effects of viscosity on the instability of viscoelastic liquid jet were shown in terms of the Ohnesorge number, and the results indicated that viscosity dampens the growth rates of instability and led to a longer breakup length.

\section{REFERENCES}

[1] Saboohi Z, Ommi F, Fakhrtabatabaei A. Development of an augmented conceptual design tool for aircraft gas turbine combustors. The International Journal of Multiphysics. 2016;10(1).

[2] RAYLEIOH L. On the instability of a cylinder of viscous liquid under capillary. 1892.

[3] Weber C. Zum zerfall eines flüssigkeitsstrahles. ZAMM-Journal of Applied Mathematics and Mechanics/Zeitschrift für Angewandte Mathematik und Mechanik. 1931;11(2):136-54.

[4] Lefebvre AH, McDonell VG. Atomization and sprays: CRC press; 2017.

[5] Lin S-P. Breakup of liquid sheets and jets: Cambridge university press; 2003.

[6] Sirignano W, Mehring C. Review of theory of distortion and disintegration of liquid streams. Progress in energy and combustion science. 2000;26(4-6):609-55.

[7] Reitz R, Bracco F. Mechanism of atomization of a liquid jet. The physics of Fluids. 1982;25(10):1730-42.

[8] Alzahabi B, Schulz K. Analysis of pressure wave dynamics in fuel rail system. The International Journal of Multiphysics. 2008; 2(3). 
[9] Squire H. Investigation of the instability of a moving liquid film. British Journal of Applied Physics. 1953;4(6):167.

[10] Hagerty W. A study of the stability of plane fluid sheets. J App Phys. 1955;22:509-14.

[11] Li X, Tankin R. On the temporal instability of a two-dimensional viscous liquid sheet. Journal of Fluid Mechanics. 1991;226:425-43.

[12] Wang X-T, Ning Z, Lü M, Sun C-H. Temporal analysis of breakup for a power law liquid jet in a swirling gas. Meccanica. 2018;53(8):2067-78.

[13] Yang L-j, Qu Y-y, Fu Q-f, Xu B-r, Zhang W, Du M-l. Linear stability analysis of a slightly viscoelastic liquid jet. Aerospace Science and Technology. 2013;28(1):249-56.

[14] Yang L-J, Tong M-X, Fu Q-F. Linear stability analysis of a three-dimensional viscoelastic liquid jet surrounded by a swirling air stream. Journal of Non-Newtonian Fluid Mechanics. 2013;191:1-13.

[15] Yang L-j, Qu Y-y, Fu Q-f, Gu B, Wang F. Linear stability analysis of a non-Newtonian liquid sheet. Journal of Propulsion and Power. 2010;26(6):1212-25.

[16] Yarin AL. Free liquid jets and films: hydrodynamics and rheology: Longman Publishing Group; 1993.

[17] Rallison JM, Hinch EJ. Instability of a high-speed submerged elastic jet. Journal of Fluid Mechanics. 1995;288:311-24.

[18] Keller JB, Rubinow S, Tu Y. Spatial instability of a jet. The physics of fluids. 1973;16(12):2052-5.

[19] Gordon M, Yerushalmi J, Shinnar R. Instability of jets of non-Newtonian fluids. Transactions of the Society of Rheology. 1973;17(2):303-24.

[20] Brenn G, Liu Z, Durst F. Linear analysis of the temporal instability of axisymmetrical non-Newtonian liquid jets. International journal of multiphase flow. 2000;26(10):162144.

[21] Liu Z, Brenn G, Durst F. Linear analysis of the instability of two-dimensional nonNewtonian liquid sheets. Journal of non-newtonian fluid mechanics. 1998;78(2-3):13366.

[22] Guo J-P, Wang Y-B, Bai F-Q, Zhang F, Du Q. Effects of Asymmetric Gas Distribution on the Instability of a Plane Power-Law Liquid Jet. Energies. 2018;11(7):1854. 
348 